\title{
Centralization of Activities in Multinational Banks, with an Application to a European Banking Group
}

\author{
Leonor S. Uva ${ }^{1} \&$ Margarida Catalão-Lopes ${ }^{2}$ \\ ${ }^{1}$ Galp Energia, Drilling-Exploration and Production Department, Lisbon, Portugal \\ ${ }^{2}$ CEG-IST, Instituto Superior Técnico, Technical University of Lisbon, Portugal \\ Correspondence: Margarida Catalão-Lopes, CEG-IST, Engineering and Management Department, Instituto \\ Superior Técnico, Technical University of Lisbon, Av. Rovisco Pais, 1049-001 Lisbon, Portugal. Tel: \\ 351-21-841-8177. E-mail: mcatalao@ist.utl.pt
}

Received: February 22, 2013

Accepted: May 3, 2013

Online Published: July 26, 2013

doi:10.5539/ijef.v5n8p136

URL: http://dx.doi.org/10.5539/ijef.v5n8p136

\begin{abstract}
Most banks operate at a multinational level. Since the diversity of geographies implies repeated operations, multinational banks are starting to centralize some functions into shared-services centres. This paper proposes a classification of the core banking areas according to their potential of centralization. As an illustration, we provide an application to a European multinational bank with important international operations. The focus is on the Card Operations Department, which is found to have high centralization potential. The results of this study show that main opportunities of centralization occur for physical card production, stock management and quality control, and fraud prevention.
\end{abstract}

Keywords: multi-domestic units, centralization potential, operational costs, efficiency, payment cards

\section{Introduction}

Technological progress facilitates the expansion of businesses across geographies. For the specific case of the banking industry, the increase in control over affiliates and the reduction in agency costs are pointed as determinants of the important geographic expansion that occurred over the past years (Berger and Deyoung, 2006). Multinational banks, however, by developing their operations across countries, with different teams and different methods, are usually faced with a duplication of operations and thus unnecessary costs. In order to avoid this situation, a possible trend is to centralize operations into one location, to achieve cost-efficiencies while taking advantage of the increased scale and cross border synergies.

However, there are issues arising from geographic diversity that pose barriers to the consolidation of operations. At a micro level, differences in language and culture across countries, as well as the existence of diverse regulatory environments, are commonly identified as the main obstacles to overcome. As we will see, they prove to be especially binding for some banking activities, in particular those which involve direct contact with clients, or dealing with procedures that are differently ruled in the various countries where the bank operates. Additionally, the potential of centralization of each banking area depends on the nature of the services delivered, meaning that only those functions whose centralization leads to added economic value, without jeopardizing quality, should be consolidated. Therefore, in order to successfully achieve the benefits of centralization, the definition of a strong strategy concerning the specifications of the countries involved, as well as the features of the areas to be merged, is required. From a macroeconomic perspective, centralization may threaten the adequate transmission of monetary policy across heterogeneous regions (e.g. Fratantoni et al., 2003), and worsen financing for small firms (Klagge \& Martin, 2005).

In this paper we propose a classification of bank operations in terms of centralization potential - high, medium or low. This taxonomy is an innovative contribution to existing literature on banking operations. As an illustration, we provide an evaluation of the potential benefits from centralization for a European banking group (Note 1). We choose an area with a high centralization potential - card operations -, and provide an analysis of the efficiency gains to be achieved by our banking group if centralization is pursued. We find that significant savings can be achieved for three sub-areas: physical card production, stock management and quality control, and fraud prevention. This paper is thus a contribution to both the literature on payment services and the literature on the 
efficiency of financial institutions (see e.g. Berger \& Humphrey, 1997 for a survey on studies addressing the efficiency of financial institutions).

As electronic payments, including card transactions, allow much lower costs than cash or paper payments, their use has become more and more widespread. This fact reinforces the importance for banks of achieving high efficiency levels in card operations. In 2010 there were approximately 1.45 payment cards per inhabitant in the European Union; card payments accounted for 39 per cent of all transactions and the ratio of paper-based to non-paper-based transactions was around one to five (http://www.ecb.int/press/pr/date/2011/html/pr110912.en.html).

As Humphrey et al. (1996) argue, shifting to electronics allows a substantial reduction in the social cost of a country's payment system, which accounts for 2 to 3 per cent of Gross Domestic Product (GDP). Debit cards, in particular, are close substitutes for cash. The incentives banks have to innovate in the payments market are addressed e.g. by Milne (2005), who provides a comparison of payments services in three Scandinavian countries, the UK and the USA. Innovation seems to have gone further in the small countries, with more concentrated banking structures, due to network effects which are stronger intra-bank than inter-bank. According to Hasan et al. (2009), the adoption of retail payment technologies actually enhances bank performance and bank stability, an aspect which may be rather important in the context of the current financial and economic crisis.

To the best of our knowledge, the current paper is the first attempt to quantify centralization gains. The existing literature on centralization is scarce (Note 2). Moreover, and in spite of the previous references, debit and credit card activities are a rather overlooked area in finance and business research. Beijnen \& Bolt (2008) is another interesting exception. The authors investigate the existence and extent of scale economies in the European payment processing industry. Their findings point to significant economies of scale for European payment processors. The same occurs in Bolt \& Humphrey (2007). These results are particularly relevant given the emergence of the Single European Payments Area (SEPA - see for instance ECB Bluebook, 2007), aimed at making cross-border payments as easy as domestic ones, besides cheaper. As Beijnen \& Bolt (2008) state, "Being cost-effective might prove to be the only viable business strategy in a competitive, intra-European payment processing market when SEPA has arrived."

The rest of the article is structured as follows. Next section brings together the distribution of banking areas according to their centralization potential, based on existing references reporting European and worldwide banks' best practices. In section 3 a brief description of the main sub-areas within the Card Operations Department is presented. Section 4 endeavours to quantify the achievable gains of operations centralization for the banking group analysed and namely in the Card Operations Department. Some conclusions and recommendations are presented in section 5. Finally, the paper ends with a section devoted to lines for future research.

\section{The Centralization Potential of Banking Areas}

Extensive know-how allied to best practices reduces activity costs. Moreover, the dimension enlargement creates the possibility of scale economies. However, not every area benefits from such an enlargement, meaning "one-size-does-not-fit-all" and, therefore, only those functions whose centralization leads to the creation of economic value and to higher control levels must be consolidated (Operations Council, 2005). The potential of centralization along banking areas can be classified as high, medium, and low or inexistent. The following subsections describe this classification in more detail.

\subsection{High Centralization Potential}

a) Call centres revealed high potential of centralization not only within the banking services, but also across other industries. Its increased importance within a company's environment, especially in terms of Customer Relationship Management (CRM) policies and of boosting cross-selling (Batt, 2002; Eichfeld et al., 2006), have led organizations to consolidate this function, so as to achieve better global results. Some of the benefits that arise are the better staff management and scheduling; the shrinkage time reduction and also the avoidance of management board duplications (Mitchell, 2001).

For the banking industry in a narrow perspective, the following distribution of the levels of centralization can be proposed, according to figure 1: i) Medium, for either operations centralization or data consolidation; ii) Intense, for the combination of the latter consolidated functions, in a virtual environment; and iii) Total, when centralization implies a physical single call centre, covering all the countries' operations, data and services (Note $3)$.

Santander is a good example of total centralization. In 2009, the Group consolidated all the call center functions into a single facility in México, where services to the clients of eight Latin American countries are delivered. 
b) Internet Banking is usually also regarded as highly central, considering the growing importance of this service within the banking industry and the similarity in the way that it is delivered to the client. The advantages found in such centralization are not only in terms of IT security systems, namely cost reductions and increased reliability (since it is cheaper and easier to audit a single facility rather than several), but also in terms of ease of adaptation to new software. ING bank centralized its internet banking systems for the eastern and central European operations (eight) into a single one in Poland and is a successful example of this strategy.

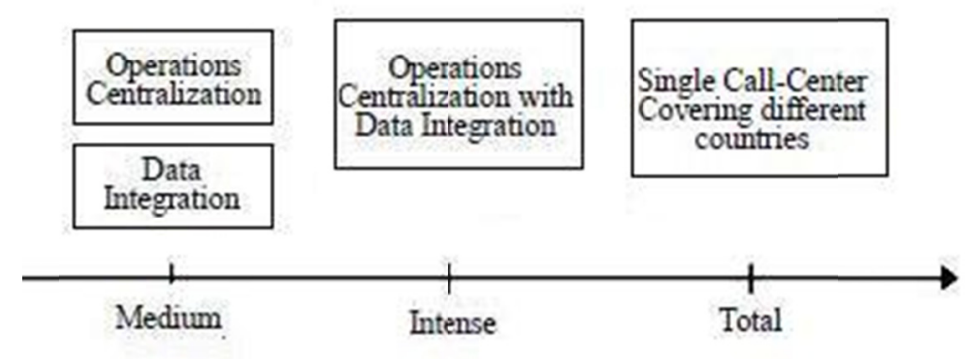

Figure 1. Degree of call centre centralization in a multinational bank

c) As far as Information Technology (IT) is concerned, evidence suggests that it also has one of the highest centralization potentials. The wide spectrum of benefits ranges from complexity reduction and increased control, to customer service improvement and integration simplification after Mergers and Acquisitions (M\&A). However, IT is run along all the banking areas and its centralization process is a very complex one. Therefore, the benefits are only achievable at the cost of some downturns like the long period of implementation (3-5 years), the training costs that stem from the staff adaptation to a new platform and, of course, the high investment costs that are related. According to Bahadur et al. (2005), the strategy defined needs to attempt to coordinate IT and senior management to guarantee the alignment between the strategic goals of the company and the technological requirements. When successfully implemented, platform integration indeed enables efficiency to improve. To prove it, there is the case of Santander's global IT platform -Parténon- responsible for improving the efficiency of Abbey Bank in 30 per cent, three years after it was acquired by Santander (Levine, 2010). BBVA and Citigroup are two other banks that already benefit from the platform integration.

d) Debit and Credit cards, also known as payment cards, reveal a high potential to be centralized due to the similarity of processes across countries, even with different portfolios. This centralization has a greater cost reduction impact on physical card production, due to eventual scale economies achieved at the producer. "The greater the volumes being processed, the lower the individual transaction cost" (Yeomans, 2004). Moreover, cost reductions are linked to the shrinkage of employee costs (both salaries and training costs). The easier global adaptation to a new development is also one of the advantages of payment cards integration. These were some of the benefits Barclays faced when it centralized the administration of the card portfolio worldwide, creating a multi-country card, to enable easier product-to-product transfers, as well as speed up new product introduction and development.

e) Human resources have been growing in importance within companies over the past decades. Therefore, banks are centralizing some of this area's activities, like training, the task consolidated at Santander's centre in Madrid. The centralization of the Human Resources data management also brings added ease in an M\&A scenario, like the Barclays case shows. After automating employee data, Barclays began handling these documents on a large scale, which was crucial by the time Woolwich Bank was integrated: the 11.000 staff files of the latter bank were automatically transferred to the Barclays' system.

f) An effective management of Purchases and Logistics can also become a competitive advantage, especially in the supply management area. Banks should try to acquire goods and services at the best total price, guaranteeing the quality and fulfilling deadlines. The registered benefits include the simplification of back office systems for supply management, easing the integration of the M\&A; and increased efficiency in choosing the right supplier through e-source based systems. On the other hand, having a decentralized supply management stems from the wish of fostering domestic economies, avoiding investment concentration in a single geographical area. Regardless of the strategy chosen, the fact is that this activity has a high centralization potential. Also within the 
Purchases and Logistics Department, evidence was found of the benefits of centralizing the management of the thousands of common documents between branches.

g) Whether in a domestic or foreign environment, all the business areas and group's activities must guarantee quality. Therefore the Quality Department has a high centralization potential since best practices, as well as standards, matrices and regulations, should be applied across countries, due to strategic concerns.

\subsection{Medium Centralization Potential}

This labelling stems from one of two reasons: i) the area apparently has potential but the best practices are still vague and limited to a small set of banks, or ii) although the area's potential is theoretically high, no evidence of banks that had centralized that area was found. The first scenario includes the payments area and mid and long term loans, while the second applies to Mobile Banking.

a) Corporate and private payment systems became complex with the banking multichannel development (internet, call centres, phone, branches, etc.), since clients can reach the bank through different means (Hunkele \& Cronenweth, 2008). The boost in the number of connections increased payment times, and real-time information became almost non-existent. To overcome customer dissatisfaction, banks started to centralize payments internally, through Service Oriented Architectures, seeking to eliminate inefficiencies. This internal centralization aligns banks across-countries for a global approach, in order to simplify international payments as well. The major hurdles that arose from this latter type of payments concern the exchange rate difference and diversity, the constant currency conversions and the dissimilar regulations. The accomplishment of SEPA aims to standardize the European regulation and eventually overcome the regulation diversity. Consequently, one can conclude that the payments area has potential of centralization across borders, but it is still unclear which implementation strategy is the best.

b) Mid and long term loans were labelled as having medium potential of centralization as well. Actually, according to the amount involved or the contract specifications, banks act differently.

c) Although Mobile Banking apparently encompasses a high centralization potential, which relies on low labour needs, centralization would only be possible through the support of centralized IT.

\subsection{Low to Non-existent Centralization Potential}

a) This is the case of branches. The majority of top executives consider branches a tactical point of contact with the client (Lippis, 2010), and so service levels must be notably high. As far as multinational banks are concerned, branches need to adapt to different requirements, inherent to customers' habits and culture, if they want to achieve high service levels. Hence, branch centralization does not seem to make much sense. Customer service-delivery differentiation is a key factor of success in banks and can only be optimized if it is managed locally, in order to be adapted to each country's culture.

Other functions revealed low potential of centralization:

b) mortgage loans, due to each country's real estate market;

c) auditing and compliance, two areas that are applied to different subjects - to the bank employees or to the bank as a whole, accordingly. Each country has different policies and regulations to be followed and therefore centralizing this department would not be rewarding.

\subsection{Synthesis}

Figure 2 presents a synthesis of the main functional areas within a bank, according to their centralization potential. In the "high" category we include the areas in which the advantages of proceeding to centralization are far greater than the disadvantages and/or obstacles, and for which there is already evidence of banks that did it. Belonging to the "medium" category can be justified by one of two reasons: either its centralizing potential exists but the best practices are still vague and limited to a few restricted set of banks (the case of payments or mid and long term loans) or, although having a high centralization potential, there is no real evidence of banks that have centralized that area/function (such as Mobile Banking for example). Finally, the areas included in "low or inexistent" category are those for which centralization, in the full meaning of the term, is not feasible.

\section{Card Business Department}

Given its high centralization potential, the payment card area is a strong candidate to be further analyzed within the framework of our banking Group, in order to detect and quantify potential efficiency gains arising from centralization among the European operations in the four countries where it operates, as well as provide a real-world illustration of the previous analysis. In this section the various subareas of the card business 
department are presented.

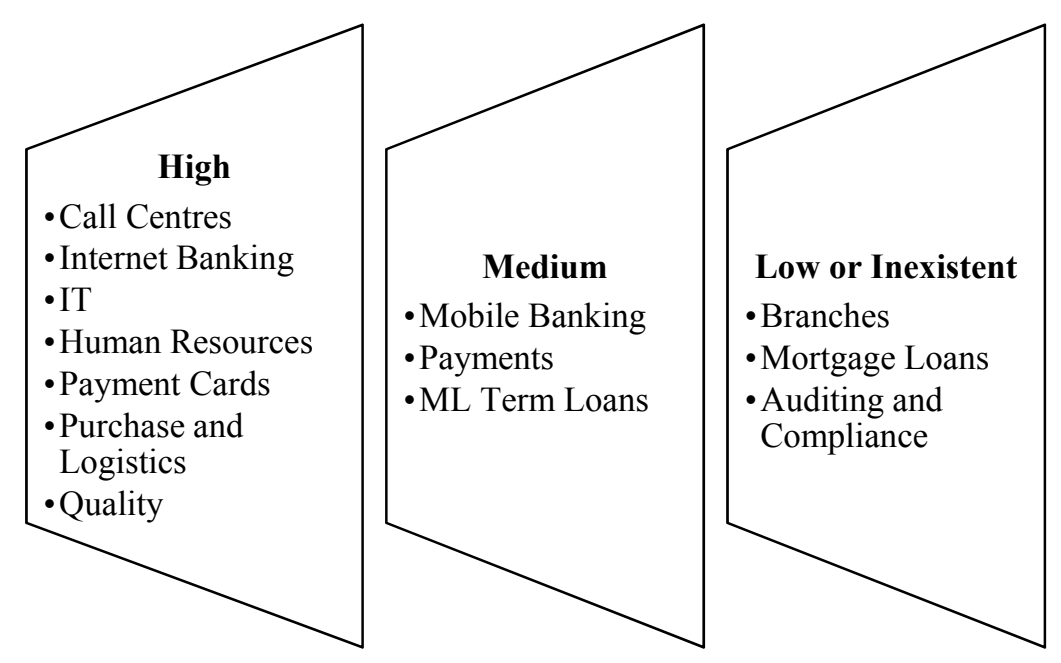

Figure 2. Labelling of banking areas, according to centralization potential

Besides being both made of plastic and used to pay for goods and services, credit and debit cards have quite distinctive features. Annual fees, grace periods or fraud protection are some of the differences between the two types of cards. Nevertheless, both types are managed similarly. At our banking Group the management is divided into the five sub-areas below, according to the different activities involved. The description that follows is useful to understand this division.

The Issuing sub-area, as the name implies, refers to the process of issuing new credit and debit cards. The processes are slightly different across countries but the core steps are the same: client's approach, credit analysis, PIN Block creation and delivery, card validation, activation, production and delivery. Three of the countries outsource their card production, while one of them opts for producing them internally.

The Pre and Post Card Production comprises stock management and quality control. The former can be either done at the outsourcer or by the bank itself. Quality control assumes three different types: i) external, when it is exclusively done at the card producer; ii) external and internal, when the bank opts to do a second analysis to guarantee the previous control done at the card processor; or iii) exclusively internal, when the card production is also internal instead of outsourced. In our Group, three countries opt for the first type, while one relies on both internal and external control.

The Servicing sub-area includes all the activities related to card functioning, namely credit limit and payment mode maintenance, card replacement, cancellation and renewal. The performance of these activities depends on the development of each platform, so some of the countries have faster services than others.

Claims Management is pursued similarly along the Group's operations - a process is triggered after a claim is received to assess responsibilities or to activate insurance. The exception is the way in which each claim is directed to the responsible area: in some countries a written document is required every time a claim cannot be handled via contact centre, while others have a central team that manages all the claims inserted in the system and just distributes them accordingly.

Finally, the Fraud Prevention sub-area is where the systems to detect fraudulent transactions are managed. The set of tools used by the bank is provided by the main international brands, including VISOR from VISA, and Riskfinder from Mastercard, among other domestic tools. These systems' functioning is based on real-time scoring, to rank transactions according to their fraudulent potential. The scoring is based on parameters like the country of origin of the card and the country where it is being used, or the transaction's amount, and each one will be treated once it gets to the top of the list - the higher the score, the greater the probability of it being a fraudulent operation. Depending on the country's regulations, the bank can either i) block the card immediately after detecting a fraudulent move, and only then contact the client; or ii) the other way around, when blocking the card is only allowed after contacting the client and, therefore, it takes longer to stop the fraudulent user. 


\section{Quantitative Analysis of the Centralization Potential in the Card Business Department}

After understanding the core of Card Operations Departments across countries, we restrict our presentation to high centralization potential sub-areas. For these we aimed at quantifying the centralization gains that may arise from such process. For the sake of comparability of the different centralization possibilities, the parameters under analysis must be standardized. Cost per employee was found to be the most adequate parameter.

The per employee cost encompasses four different cost types: i) Payments, that refers to wages, bonus and other monetary benefits; ii) Physical Space, that, as the name implies, comprises the costs spent with the physical space per employee; iii) IT and Communication, that include those costs with technological equipment (internet, applications, phone, software, etc.); and iv) Others, that refer to a wide spectrum of expenses, from office materials to cleaning products.

Since each employee is usually a multi tasked individual, it is difficult to measure the exact time that each one spends in each activity. Therefore, the cost per FTE - Full Time Equivalent - parameter was used, to determine the exact allocation of costs within each sub-area. FTEs measure the contribution of an employee working in full time (eight hours per day) for a specific task. In our Group the relative allocation of FTEs is done as presented in table 1 .

Table 1. Relative distribution of FTEs across sub-areas (2008)

\begin{tabular}{lcccc}
\hline & Country A & Country B & Country C & Country D \\
\hline Issuing & $4.4 \%$ & $13.4 \%$ & $1.9 \%$ & $8.3 \%$ \\
Servicing & $2.4 \%$ & $10.4 \%$ & $1.2 \%$ & $1.3 \%$ \\
Card production & $1.6 \%$ & $11.9 \%$ & $0.1 \%$ & $0.7 \%$ \\
Claims Management & $19.2 \%$ & $4.4 \%$ & $3.6 \%$ & $2.8 \%$ \\
Fraud Prevention & $5.2 \%$ & $5.9 \%$ & $0.6 \%$ & $0.7 \%$ \\
Global & $32.7 \%$ & $45.9 \%$ & $7.4 \%$ & $13.9 \%$ \\
\hline
\end{tabular}

\subsection{Card Production and Delivery}

Although this activity is commonly included in the Issuing sub-area (because of the inherent need to produce a card in every issuing process), one must take into consideration that it also involves the Servicing sub-area, in terms of replacements and renewals. Therefore, we considered the card production and delivery costs as the ones imputed to new cards, to renewals and to replacements.

In order to obtain global costs in each country (GCi), different approaches were used, according to the type of production. When it is outsourced, global card production costs depend on the card prices charged by the outsourcer for credit and debit cards $-\mathrm{p}_{\mathrm{cc}}$ and $\mathrm{p}_{\mathrm{dc}}-$ and on the volume of new credit and debit cards produced $-\mathrm{v}_{\mathrm{ncc}}$ and $\mathrm{v}_{\mathrm{ndc}}$. These variables are related through equation 1 , for country $\mathrm{i}$ :

$$
G C i=\sum\left(p_{c c, i} * v_{n c, i}+p_{d c, i} * v_{n d c, i}\right)
$$

In turn, if card production is internal to the bank, i.e., no external processors intervene, GCi depend on in-house costs such as: embossing machine depreciation $\left(\mathrm{D}_{\mathrm{m}}\right)$, cost of plastics and personalization $\left(\mathrm{C}_{\mathrm{pp}}\right)$, costs with card delivery $\left(\mathrm{C}_{\mathrm{d}}\right)$ and costs with mail and marketing materials $\left(\mathrm{C}_{\mathrm{mm}}\right)$. In this case, global production costs $(\mathrm{GCi})$ are given by equation (2), for country $\mathrm{i}$ :

$$
G C i=\sum\left(D_{m}+C_{p p}+C_{d}+C_{m m}\right)
$$

The global card production and delivery costs reveal a distribution of 59 per cent in terms of issuing (new cards) and 41 per cent concerning servicing (renewals and replacements).

We want to analyze the impacts of centralizing the card production activity along our Group's European operations - countries A, B, C and D. Such consolidation encompasses the merging of the Group's five outsourcers (in country A the Group has two outsourcers) into a single one. However, since choosing the central outsourcer location is a cost-related decision, the added transportation needs of sending cards across countries must be taken into consideration. In fact, the major downsize of having a central outsourcer in country A is the impact on delivery cost and delivery time for countries B, C and D. The former disadvantage can be diminished if the increase in delivery costs does not exceed the cost reductions gained with scale economies; the latter can only be surpassed so far: some cards should be issued locally to fulfil the urgent requests. To overcome the 
hurdle of urgent requests (that represent approximately 4 per cent of the global card production) we propose a mix of central and domestic card production, in a 96 per cent -4 per cent proportion.

The proposal implies some benefits and disadvantages from which the following should be enhanced:

a) Decrease in the unit card price charged by the central outsourcer located in country A, since it would benefit from a volume increase of 96 per cent of the global production in countries B, C and D. The current outsourcers were invited to tender for the discounts they would make if centralization was in the outsourcer's country, according to the new volume of production for the case when centralization is in each country. The offers considered are presented in table 2.

Table 2. Outsourcers discount offers (\%)

\begin{tabular}{cccccc}
\hline Outsourcer & $\mathrm{A}_{1}$ & $\mathrm{~A}_{2}$ & $\mathrm{~B}$ & $\mathrm{C}$ & $\mathrm{D}$ \\
\hline Discount (\%) & 17.5 & 19 & 15 & 17.2 & 15 \\
\hline
\end{tabular}

b) Increase of the unit card price charged by the domestic outsourcers, since each would pass from a volume production of 100 per cent to 4 per cent. According to our Group Experts', this would imply a duplication (+100 per cent) of the current unit cost;

c) Added transportation costs linked to the need of sending, via international mail, all the cards produced centrally with destination other than the country where the central outsourcer is located. International mail costs (measured in $€ / \mathrm{kg}$ ) are higher than national's.

From the analysis of the volumes of cards produced in each country, the expected result is for the central outsourcer to be located in our Group's headquarters (country A), since it has the highest volume of cards produced. Therefore, centralizing production in any other country would imply the international transport of 96 per cent of that volume (plus 96 per cent of the other two countries' volume), leading to high transportation costs. Moreover according to the outsourcers' proposals it is rational to say that the chosen outsourcer is likely to be $\mathrm{A}_{2}$ since it offered a more competitive unit cost reduction when compared to $A_{1}$ (19 per cent vs. 17.5 per cent), or even compared to any of the others. Nevertheless, an exhaustive analysis was done to quantify exactly the cost reductions achievable, based on information on current prices and volumes in each country, as well as estimated transportation costs and proposed discounts. For each of the eligible outsourcers, the results are presented in table 3.

Table 3. Savings achievable due to operations centralization (\%)

\begin{tabular}{cccccc}
\hline Outsourcer & $\mathrm{A}_{1}$ & $\mathrm{~A}_{2}$ & $\mathrm{~B}$ & $\mathrm{C}$ & $\mathrm{D}$ \\
\hline Reductions Achievable (\%) & 10 & 11.5 & 6.3 & 3.5 & -3.8 \\
\hline
\end{tabular}

From table 3 we can conclude that centralizing operations would be most valid if the outsourcer is located in country A, since centralization in any of the other countries would lead to almost no cost reductions or even to a cost increase (country D). These results corroborate the previous expectations and stem mainly from the difference of card volumes to be produced: although unit transportation costs (in EUR per kg of cards) are higher for the other countries involved, the volume of cards to be sent to headquarters is so high that overcomes that difference of prices. Moreover, the unit prices of this country's outsourcers $\left(A_{1}\right.$ and $\left.A_{2}\right)$ are more competitive than the ones abroad.

Summing up, the most profitable option is to centralize 96 per cent of the global production in a single country at outsourcer $\mathrm{A}_{2}$, with which the Group can achieve a reduction of 11.5 per cent in the global card production and delivery costs. This country's card volume (and 96 per cent of the volume of the other countries) should be produced at outsourcer $\mathrm{A}_{2}$. The 4 per cent left of each country's card volume should be produced at domestic outsourcers, namely at $\mathrm{B}, \mathrm{C}$ and $\mathrm{D}$.

Regarding outsourcers $A_{1}$ and $A_{2}$ results, it is also interesting to discuss its slight difference from a competition perspective. This difference is clearly justified by the more competitive proposal presented by outsourcer $\mathrm{A}_{2}(19$ per cent vs. 17.5 per cent) in terms of the total cost reduction achievable. These outsourcers' proposals, however, 
were done without one being aware of the other's, and in a merely informative context. If that was not the case, a competitive environment could be triggered between outsourcers and, in the limit, we would have a sort of a Bertrand paradox, with these two duopolists decreasing price until marginal cost. In this situation the client (the banking Group, in this case) would be the winner. This is especially relevant given that having a single supplier represents a loss of bargaining power and hence a potential profitability reduction.

\subsection{Pre and Post Card Production}

As far as stock management and quality control are concerned, their execution can be done either internally or in outsourcing. In the former, deciding upon either method can be controversial since one's benefits are the other's disadvantages: internal stock management enables access to more accurate information in terms of products, while having this activity outsourced frees bank employees for their core functions and the stock management is executed by those who are more informed on the best methods and practices - the producers. Within the framework of a central outsourcer, we support the advantages of external stock management and recommend its centralization. Each bank only needs to ensure that the file with the volume of cards to be issued is produced under the same template across countries, to avoid having different file models.

In turn, although quality control can be either internal or external, we support the latter, considering that the outsourcer has the capacity to perform quality control on all the cards produced, while internal control is limited to a small number of verifications that are not indexed to the global volume of cards produced. Therefore, and also as a consequence of the outsourcers' consolidation, we propose the centralization of the quality control activity.

\subsection{Fraud Prevention}

Fraud is a global threat. Aware of this reality, the Group is developing a fraud prevention centralization project that aims to consolidate this activity for its European operations. The project is based on a common platform whose functioning is similar to VISOR's, with real time scoring of transactions. However, the Group's common platform brings a new set of advantages that may overcome some of the main barriers in a centralization process. From the advantages, the following should be highlighted: i) collecting the information from different systems in different languages, to then present it in English; ii) having the full workload available on the screen, instead of having only the highest scored transaction - this allows better information regarding the number of FTEs needed to fulfil the daily workload, that otherwise is impossible; iii) having support $24 / 7$, which increases fraud detection out of hours and respects each time zone and iv) since a common platform defines standard procedures across countries, the best practices will be applied everywhere.

The theoretical benefits are evident; however, we intended to quantify the achievable gains. The actual global fraud costs encompasses the following parameters and respective index values: i) the costs with FTEs allocated to fraud prevention across operations (6); ii) the current costs with the software licenses (23) and iii) the global fraud volume, referring to the amount associated to the fraudulent transactions (71). Hence the current global fraud costs at the Group are 100, in index values.

Since we intend to identify the impact of (i) having a centralized team (ii) working in a single platform, (iii) with higher effectiveness, we need to quantify (a) the post centralization FTEs costs, (b) the new platform software license price and (c) the global fraud volume with increased effectiveness. As some of these parameters, namely (a) and (c), are not yet certain, a sensitivity analysis was done, for different scenarios.

Concerning (a), since the exact number of FTEs required for the central team is not yet known, neither are the FTEs costs. We therefore assumed three scenarios, where the base structure is the sum of the current FTEs allocated to fraud prevention across countries, and the other two refer to a positive and negative variation of two FTEs. Concerning (c), the global expectable fraud volume is hard to measure since the platform's increased effectiveness is not certain until it is actually implemented or a Prototype Test is done - the platform analyzes the same set of transactions that the operations once had during the same period of time, to measure the difference of fraudulent transactions detected. Due to such uncertainty, we considered four different possibilities of increased effectiveness ( 5 per cent, 10 per cent, 15 per cent and 20 per cent) and an extra possibility of a 22 per cent lower effectiveness to understand the limits of feasibility of this project. The sensitivity analysis results are presented in table 4. 
Table 4. Sensitivity analysis to the future global fraud costs (in index values)

\begin{tabular}{cccc}
\hline FTEs & Plus 2 & Base Structure & Less 2 \\
Effectiveness & & 79 & 77 \\
\hline Unlikely $(+5 \%)$ & 81 & 75 & 73 \\
Weak $(+10 \%)$ & 77 & 72 & 70 \\
Satisfactory (+15\%) & 74 & 68 & 66 \\
Optimistic $(+20 \%)$ & 70 & 98 & 96 \\
$-22 \%$ & 100 & &
\end{tabular}

From the sensitivity analysis one can conclude that implementing the new platform will always be profitable to the Group even in the unlikely scenario where its effectiveness is only 5 per cent greater than the present systems and required FTEs increase by two-global fraud costs will decrease 19 per cent. However, in the best case scenario-20 per cent effectiveness increase and a central team with less 2 FTEs-cost reductions can achieve up to 34 per cent.

The fraud prevention centralization project would only not be feasible if the new platform detects 22 per cent less fraud volume than the actual systems and if the central team requires two more FTEs. In that case, the gains will be zero, since the new global fraud costs would be 100 as well.

\section{Conclusions and Implications}

In order to achieve significant cost reductions and hence higher efficiency levels to remain competitive, multinational banks are improving their management practices and pursuing a growing trend of operations' centralization across countries. This article aimed firstly to identify the centralization potential of the banking areas, based on the best practices of European and worldwide banks. Call centres, internet banking, IT, human resources, payment cards, purchase and logistics, and quality were found to have the highest centralization potential. Afterwards, a specific application was done to a European banking group, focused on the centralization of the payment card area, along the Group's European operations. Results showed that the Group can achieve up to 11.5 per cent card production and delivery cost reduction, if this centralization occurs. Pre and post card production activities should also be centralized. Regarding fraud prevention, the activity consolidation into a single platform can lead the Group to a global fraud volume reduction of 34 per cent, if the best case scenario is accomplished.

To the best of our knowledge, this was the first attempt made in the literature to quantify centralization gains. The present paper demonstrated that there may be room for improved efficiency in the banking sector, particularly for those institutions that operate in multiple geographies, provided that culture and regulation asymmetries are not a too serious obstacle. Clearly, an immediate managerial implication of our results is that multinational banks may be able to improve their efficiency ratios and hence their competitiveness in the global market by simply centralizing some multi-domestic activities. More efficiency means less need for financing and improved solvability ratios, which may be particularly important in times of economic and financial crisis. In the current adverse macroeconomic scenario, the gains achieved in fraud prevention may be quite relevant for those institutions more severely faced with overdue credit.

From a theoretical perspective, this paper calls the attention to a somewhat new competition variable, the degree of centralization. We expect investments in centralization to be strategic complements, in the sense that increased centralization by a given institution triggers a new or an additional centralization effort by rivals (Fudenberg \& Tirole, 1984). This leads to overall efficiency gains, the possibility of lower prices and improved welfare. Although we concentrated our analysis on the banks' perspective, it seems that there exist positive externalities associated with an institution's centralization effort.

\section{Limitations and Lines for Future Research}

Future investigation on this topic should consider the competition issue. To start with, an identical study could be conducted for those banking groups that directly compete with the one being analysed here, in order to compare achievable centralization gains. If they are of similar magnitude, one can expect competition to be fiercer with respect to this variable than if they substantially differ and/or are almost irrelevant for one of the competitors. 
Taking for instance two extreme samples, one including the top 10 European banks (the largest) as compared with the bottom 10, could also allow some interesting insights.

Importantly too, and since this paper was mainly focused on centralization advantages, future research should try to evaluate possible losses from centralization, namely those arising from differences in language, culture or regulation, to be balanced with quantified gains in banking areas where advantages and disadvantages do coexist. Another relevant extension would consider evaluating increased banks' efficiency benefits to consumers and to society as a whole. Finally, future research could also, of course, be extended to other industries-financial and nonfinancial-with multi-domestic operations, in order to compare sectors in terms of potential efficiency gains.

\section{References}

Bahadur, K., Desmet, D., \& Bommel, E. V. (2005). Smart IT spending: Insights from European banks. McKinsey on IT, Innovations in IT Management, 6. McKinsey \& Company.

Batt, R. (2002). Managing customer services: Human resource practices, quit rates and sales growth. Academy of Management Journal, 45(3), 587-597. http://dx.doi.org/10.2307/3069383

Beijnen, C., \& Bolt, W. (2009). Size matters: Economies of scale in European payments processing. Journal of Banking \& Finance, 33(2), 203-210. http://dx.doi.org/10.1016/j.jbankfin.2008.07.014

Berger, A. N., \& Deyoung, R. (2006). Technological progress and the geographic expansion of the banking industry. Journal of Money, Banking and Credit, 38, 1483-1513. http://dx.doi.org/10.1353/mcb.2006.0077

Berger, A. N., \& Humphrey, D. B. (1997). Efficiency of financial institutions: International survey and directions for future research. European Journal of Operational Research, 98, 175-212. http://dx.doi.org/10.1016/S0377-2217(96)00342-6

Bolt, W., \& Humphrey, D. B. (2007). Payment network scale economies, SEPA, and cash replacement. Research Department, Federal Reserve Bank of Philadelphia, Working paper.

ECB. (2007). Payment and securities settlement systems in the European Union (4th ed.). Blue Book, Vol.1: euro area countries.

Eichfeld, A., Morse, T. D., \& Scott, K. W. (2006). Using call centres to boost revenue. McKinsey on Banking. The Mckinsey Quarterly: McKinsey \& Company.

Fratantoni, M., \& Schuh, S. (2003). Monetary policy, housing and heterogeneous regional markets. Journal of Money, Banking and Credit, 35, 557-589. http://dx.doi.org/10.1353/mcb.2003.0029

Fudenberg, D., \& Tirole, J. (1984). The Fat-cat effect, the Puppy-dog ploy, and the lean and hungry look. The American Economic Review, 74, 361-366.

Humphrey, D. B., Pulley, L. B., \& Vesala, J. M. (1996). Cash, paper, and electronic payments: A cross-country analysis. Journal of Money, Banking and Credit, 28, 914-939. http://dx.doi.org/10.2307/2077928

Hunkele, D., \& Cronenweth, E. A. (2008). Centralizing wholesale payment services: The key to improved Growth, efficiency and risk management. Finsights, Technology Insights for the Financial Services Industry, ch.7.

Hunt, R. (2009). Case study: State bank of India. World's Largest Centralized Core Processing Implementation. The Tower Group, Inc.

Klagge, B., \& Martin, R. (2005). Decentralized versus centralized financial systems: Is there a case for local capital markets? Journal of Economic Geography, 5, 387-421. http://dx.doi.org/10.1093/jeg/lbh071

Levine, J. (2010). Santander: The most boring bank in the world. Retrieved from http://www.time.com/time/magazine/article/0,9171,1951914-2,00.html\#ixzz0muTbCdsl

Lippis, N. J. (2010). A new era in borderless branch experience emerges: are you ready? Lippis Consulting.

Milne, A. (2005). What's in it for us? Network effects and bank payment innovation. Bank of Finland Research, Discussion Papers.

Mitchell, I. (2001, December). Call center consolidation-Does it still make sense? Business Communications Review, 24-28.

Operations Council. (2005). Unchartered territory: from organization structure to operations solutions.

Yeomans, J. (2004). Centralization-The route to controlling payment costs. Card Technology Today, 16(5), 1011. http://dx.doi.org/10.1016/S0965-2590(04)00103-3 


\section{Notes}

Note 1. For confidentiality reasons, the name of the Group is not mentioned and all the information that could enable identification has been suppressed throughout the paper.

Note 2. Hunt (2009) presents a case study on the implementation of a centralized core system by the State Bank of India. Finansbank, a Turkish bank, centralized more than 65 per cent of its operation transactions in a platform called FOMER and intends to centralize the entirety, except for those that can only be provided by the branches, such as counter and similar transactions (http://www.finansbank.com.tr/en/about-finansbank/departments/operations.aspx).

Note 3. The financial crisis that emerged in 2007-2008 calls for a critical view on CRM and cross-selling in banking, taking into account the miss-selling and predatory lending facilitated by financial services marketing.

\section{Copyrights}

Copyright for this article is retained by the author(s), with first publication rights granted to the journal.

This is an open-access article distributed under the terms and conditions of the Creative Commons Attribution license (http://creativecommons.org/licenses/by/3.0/). 